\title{
Cardiac disease in pregnancy: still an arduous conundrum for the obstetrician
}

\author{
Sheeba Marwah ${ }^{1}$, Manjula Sharma ${ }^{1}$, Harsha Shailesh Gaikwad ${ }^{1}, \operatorname{Ritin}_{\text {Mohindra }}{ }^{2}$ \\ ${ }^{1}$ Department of Obstetrics and Gynaecology, Vardhaman Mahavir Medical College and Safdarjung Hospital, New \\ Delhi, India \\ ${ }^{2}$ Department of Internal Medicine, Vardhaman Mahavir Medical College and Safdarjung Hospital, New Delhi, India
}

Received: 29 February 2016

Accepted: 07 April 2016

\section{*Correspondence:}

Dr. Sheeba Marwah,

E-mail: sheebamarwah@yahoo.co.in

Copyright: (C) the author(s), publisher and licensee Medip Academy. This is an open-access article distributed under the terms of the Creative Commons Attribution Non-Commercial License, which permits unrestricted non-commercial use, distribution, and reproduction in any medium, provided the original work is properly cited.

\section{ABSTRACT}

Cardiac diseases, complicating about 1 percent of all pregnancies, account for significant maternal morbidity and mortality by being the leading cause of obstetrical intensive care unit admissions and of indirect maternal deaths. Of late, there has been observed a changing pattern in heart disease, the etiology having shifted from primarily rheumatic to predominantly congenital (75-82\%), with shunt lesions preponderating (20-65\%). Counseling and management of women of childbearing age with suspected cardiac disease ought to commence prior to conception; they should be managed by interdisciplinary teams; high risk patients must be treated in specialized facilities, and diagnostic procedures and interventions should be executed by mavens with profound expertise in the cardiovascular diseases and proficiency in treating pregnant women. This article provides a comprehensive review on management of cardiac disease in pregnancy to assist obstetricians in tackling this mystifying medical situation effortlessly, attaining a favorable feto-maternal outcome.

Keywords: Cardiac disease in pregnancy, Management

\section{INTRODUCTION}

Adequate erudition of heart disease in pregnancy is imperative to the obstetrician because it is the $4^{\text {th }}$ chief cause, and foremost indirect cause of maternal mortality, responsible for $0.5-2.7 \%$ of maternal deaths. Its incidence is $\sim 1 \%$ of pregnancies $\left(0.1 \%\right.$ to $4 \%$.). ${ }^{1}$ Advances in the medical and surgical treatment of patients with congenital heart defects have ensued in an increased subsistence to reproductive age. Also better access to medical facilities, utilization of new-fangled antibiotics, besides enhanced surgical techniques have led to a diminution in the incidence from $0.9 \%$ to $0.3 \%$ of rheumatic heart disorders (RHD), especially in industrialized countries. ${ }^{2}$ Nevertheless, in developing countries, RHD still continues to form the major encumber of heart disease $(56-89 \%)^{3,4}$. Cardiomyopathies are infrequent, but exemplify severe causes of cardiovascular complications in pregnancy, peri-partum cardiomyopathy (PPCM) being the most frequent. ${ }^{5}$

\section{Hemodynamic changes in pregnancy}

\section{Antenatal changes}

Plasma volume attains a maximum of $40 \%$ above baseline at 24 weeks gestation. ${ }^{6}$ A $30-50 \%$ upsurge in cardiac output (CO) befalls in normal pregnancy. In early pregnancy increased $\mathrm{CO}$ is primarily associated with rise in stroke volume; however, later, heart rate is the major contributory factor. Heart rate (HR) begins to rise at 20 weeks and increases until 32 weeks, the rise persists 2-5 days postpartum. Systemic BP (SBP) typically falls early in gestation and diastolic BP (DBP) is usually $10 \mathrm{mmHg}$ 
below baseline in the second trimester. This decrease in BP is caused by active vasodilatation, achieved through the action of local mediators such as prostacyclin and nitric oxide. In the third trimester, the DBP gradually increases and may normalize to non-pregnant values by term (Figure 1).

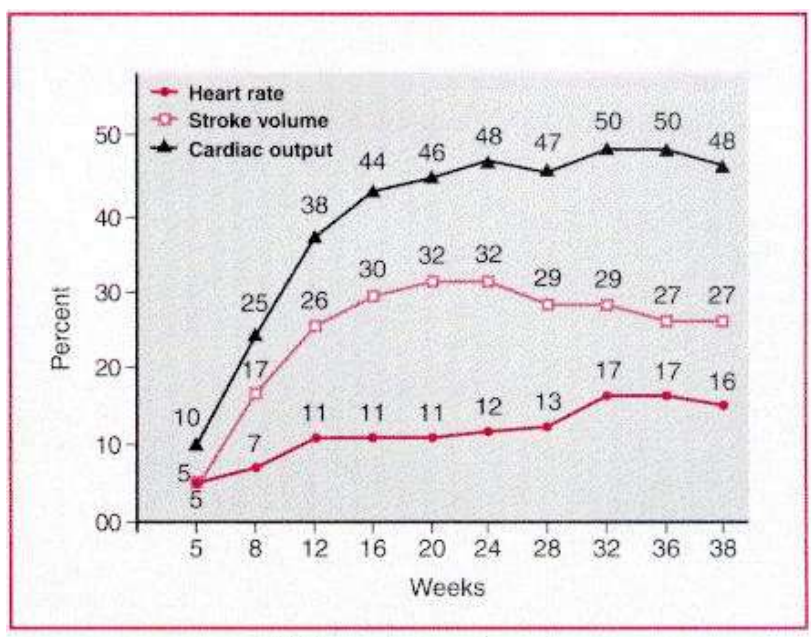

Figure 1: Hemodynamic changes in pregnancy.

\section{Intrapartum changes}

The pain and anxiety of labor leads to increase in HR leading to increased afterload. ${ }^{7}$ The amplified catecholamines also contribute to augmented cardiac load. Each uterine contraction further causes centralization of 300-500cc of blood from utero-placental circulation.

\section{Postpartum changes}

Cardiac Output-Immediately post-delivery, CO increases further by $30 \%$ for a brief period. This is due to loss of utero-placental shunt; relief of venacaval compression by gravid uterus; auto-transfusion of utero-placental blood and decreased colloid oncotic pressure. Within 1 hour, $\mathrm{CO}$ and $\mathrm{HR}$ return to third trimester values, whereas MBP and SV return by 24 hours post-delivery. All hemodynamic changes begin to reverse within 1-3days, and gradually return to pre-pregnancy values within 12 to 24 weeks after delivery.

\section{Normal physiological changes of pregnancy that mimic cardiac disease ${ }^{8}$}

Symptoms: Tiredness, Dyspnoea, Orthopnoea, Syncope, Light-headedness, Decreased exercise capacity

Physical signs: Faster resting HR, peripheral oedema, hyperventilation, bounding pulses, distended neck veins, loud S1, soft Grade I and II systolic murmurs (best heard at upper left sternal edge or pulmonary area in $96 \%$ of pregnant women, believed to be caused by increased flow across the aortic and pulmonary valves), cervical venous hum, mammary souffle.

Electrocardiogram: Left axis deviation, ST segment changes, small Q, inverted P wave in lead III abolished by inspiration, $\mathrm{T}$ wave inversion in lead III, atrial or ventricular ectopics, Right bundle branch block (RBBB).

Chest X-ray: Apparent cardiomegaly (elevation of diaphragm), straightened left upper cardiac border, horizontal heart position, enlarged L.A. (lateral views), increased vascular lung markings, small early postpartum pleural effusions (right sided).

Echocardiogram: Increased left/right ventricular dimensions, functional tricuspid/pulmonary insufficiency (94\% at term), Trivial TR (43\%), trivial MR (28\%) known as physiological MR, small pericardial effusions ( $40 \%$ in postpartum period).

\section{When should an obstetrician suspect heart disease in a pregnant woman?}

Symptoms: Progressive severe dyspnea/orthopnea, nocturnal cough, hemoptysis, exertional syncope, exertional chest pain, progressive edema/anasarca, tachycardia $120 \mathrm{bpm}$, any s/s at rest, $\mathrm{H} / \mathrm{O}$ failure in past or current pregnancy. ${ }^{8}$

Physical signs: Cyanosis ,clubbing, persistent neck vein distension, persistent systolic murmurs (grade 3/6), diastolic murmurs, unequivocal enlargement of heart on CXR, heave, persistent arrhythmias (atrial fibrillation/flutter), persistent split in S2, pulmonary hypertension, murmurs associated with stenotic lesions accentuated (due to increased blood volume and $\mathrm{CO}$ ), murmurs of AR, MR, VSD may become attenuated (due to decrease in SVR). ${ }^{8}$

\section{Investigations}

a. ECG and Chest X-ray if indicated. ${ }^{9}$

b. 24-hour Holter monitoring may be required to diagnose arrhythmias.

c. Echocardiography is non-invasive and safe. With Mmode, 2D and Doppler capabilities, CHD is detected and cardiac functional status assessed. Transoesophageal echocardiography (TOE) is useful in selected cases, e.g. Assessment of Infective Endocarditis (IE), aortic dissection, or a technically difficult transthoracic study. ${ }^{10,11}$

d. Magnetic resonance cardiac imaging is useful when other modalities fail. However, safety in pregnancy and effects on foetus has not been established. ${ }^{12-15}$

e. Radionuclide cardiac imaging and Left Heart Catheterization, not recommended in pregnancy.

\section{Management of heart disease in pregnancy}

\section{Individualization of management}


2. Multidisciplinary approach (obstetrician, cardiologist, anesthetist, neonatologist, pediatric cardiologist and if appropriate, the cardiothoracic surgeon). ${ }^{16,17}$

3. Risk assessment Risk stratification should be based on cardiac lesion, baseline functional status, possibility and probability of cardiac complications (Table 1, 2 and 3). ${ }^{2,4,18}$

Table 1: Siu risk Score 2004 (NOPE) Predictors of maternal cardiovascular events. ${ }^{4}$

Baseline NYHA functional class $>$ II or cyanosis.

Left heart Obstruction (mitral valve area $<2 \mathrm{~cm} 2$, aortic valve area $<1.5 \mathrm{~cm} 2$, peak LV outflow tract gradient $>30 \mathrm{mmHg}$ byechocardiography).

Prior cardiac event (heart failure, transient ischemic attack, stroke before pregnancy or arrhythmia).

Reduced systemic ventricular systolic function (Ejection fraction $<40 \%$ ).

CARPREG risk score: for each CARPREG predictor that is present a point is assigned. 0 point $-5 \%, 1$ point $-27 \%, 1$ point $75 \%$.

Table 2: NYHA functional classification.

\begin{tabular}{|c|c|}
\hline \multirow{2}{*}{ Class I } & $\begin{array}{l}\text { No functional limitation of } \\
\text { activity }\end{array}$ \\
\hline & $\begin{array}{l}\text { No symptoms of cardiac } \\
\text { decompensation with activity. }\end{array}$ \\
\hline \multirow{3}{*}{ Class II } & $\begin{array}{l}\text { Mild amount of functional } \\
\text { limitation. }\end{array}$ \\
\hline & Patients are asymptomatic at rest. \\
\hline & $\begin{array}{l}\text { Ordinary physical activity results in } \\
\text { symptoms. }\end{array}$ \\
\hline \multirow{3}{*}{ Class III } & Limitation of most physical activity. \\
\hline & Asymptomatic at rest \\
\hline & $\begin{array}{l}\text { Minimal physical activity results in } \\
\text { symptoms. }\end{array}$ \\
\hline \multirow{2}{*}{ Class IV } & $\begin{array}{l}\text { Severe limitation of physical } \\
\text { activity results in symptoms. }\end{array}$ \\
\hline & $\begin{array}{l}\text { Patients may be symptomatic at } \\
\text { rest/heart failure at any point of } \\
\text { pregnancy. }\end{array}$ \\
\hline
\end{tabular}

4. Principles of Pre-conceptional care

a. Multidisciplinary approach

b. Counseling patients regarding

- $\quad$ Risk assessment

- Any contraindication to pregnancy

- Discontinuation of teratogenic drugs like ACE inhibitors and oral anticoagulants.
Table 3: Modified WHO classification of maternal cardiovascular risk. ${ }^{18}$

Conditions in which pregnancy risk is WHO I

Uncomplicated, small or mild

Pulmonary stenosis

Patent ductus arteriosus

Mitral valve prolapse

Successfully repaired simple lesions (atrial or ventricular septaldefect, patent ductus arteriosus, anomalous

pulmonary venousdrainage).

Atrial or ventricular ectopic beats, isolated

Conditions in which pregnancy risk is WHO II or III

WHO II (if otherwise well and uncomplicated)

Unoperated atrial or ventricular septal defect

Repaired tetralogy of Fallot

Most arrhythmias

WHO II-III (depending on individual)

Mild left ventricular impairment

Hypertrophic cardiomyopathy

Native or tissue valvular heart disease not considered

WHO I or IV

Marfan syndrome without aortic dilatation

Aorta $<45 \mathrm{~mm}$ in aortic disease associated with bicuspid aortic valve

Repaired coarctation

WHO III (pregnancy very high risk)

Mechanical valve

Systemic right ventricle

Fontan circulation

Cyanotic heart disease (unrepaired)

Other complex congenital heart disease

Aortic dilatation 40-45 $\mathrm{mm}$ in Marfan syndrome

Aortic dilatation $45-50 \mathrm{~mm}$ in aortic disease associated with bicuspidaortic valve

Conditions in which pregnancy risk is WHO

IV(pregnancy contraindicated)

Pulmonary arterial hypertension of any cause

Severe systemic ventricular dysfunction (LVEF $<30 \%$, NYHA III-IV)

Previous peripartum cardiomyopathy with any residual impairment ofleft ventricular function

Severe mitral stenosis, severe symptomatic aortic stenosis

Marfan syndrome with aorta dilated $>45 \mathrm{~mm}$

Aortic dilatation $>50 \mathrm{~mm}$ in aortic disease associated with bicuspidaortic valve

Native severe coarctation

5. Principles of Antenatal care

a. Look for occurrence of new symptoms or worsening of pre-existing ones like fever, breathlessness, cough, chest pain, palpitations during each antenatal visit.

b. Women with uncomplicated cardiac disease should be seen once month till 28-30weeks, two weekly till 36 weeks, weekly thereafter till delivery, or earlier as and when required 
c. Fe and FA, calcium supplementation as tolerated.

d. Any additional investigation as advised by cardiologist.

e. Foetal surveillance including USG for FWB, Foetal ECHO in women with CHD.

f. Hospital Admission-NYHA I, II-36 weeks, NYHA III, IV-at first visit.

g. Identification and management of co-morbidities.

h. Identification of S/S of decompensation such as easy fatigability, dysponea,orthopnea/paroxysmal nocturnal dyspnea/nocturnal cough/hemoptysis/tachycardia/S 3 gallop/basal crepitation.

6. Intrapartum care

a. Cervical ripening can be done with prostaglandins.

b. Vaginal delivery is preferred unless contraindicated. $^{19}$

c. Indications of LSCS are

- Moderate/Severe MS in NYHA III/IV, or with PAH.

- Severe Aortic stenosis.

- Pulmonary hypertension (Primary or secondary).

- Risk of Aortic Dissection-Marfan Syndrome with dilated aortic root dilatation $>40 \mathrm{~mm}$ and coarctation of aorta.

- Mother on oral anticoagulants in labor.

- Obstetric indications.

\section{Ist stage of labor}

1. Good IV access, I/O record, should be optimally hydrated with oral clear liquids during labor. IV fluids are given if required at rate not exceeding $75 \mathrm{ml} / \mathrm{hr}$ (15drops/min) except in fixed CO lesions (AS, hypertrophic sub-aortic stenosis) or bidirectional shunt lesions like ASD, VSD, and PDA where fluids are given at the rate of $125 \mathrm{ml} / \mathrm{hr}$ (30 drops / $\mathrm{min})$.

2. Semi recumbent with left/right lateral tilt is the preferred position as it lessens the hemodynamic fluctuations associated with contractions when the patient is supine.

3. Vitals monitoring and frequent chest auscultations for basal crepts must be done; PR>100bpm, RR>24 S/o impending failure.

4. Humidified oxygen to avoid nasal irritation should be given.

\section{Foetal HR monitoring during labor.}

6. Pain Relief, Epidural is best (contraindicated in Eisenmenger's, AS, PAH, intracardiac shunts where parenteral narcotics with pudendal block are preferred).

7. Limited $\mathrm{P} / \mathrm{V}$ examinations to be done under strict aseptic precautions.
8. I E Prophylaxis should be given as per recommendations 20 (Table 4 and 5).

9. Augmentation of labor should be done with concentrated oxytocin drip $<75 \mathrm{ml} /$ hour.

Table 4: Cardiac Conditions with high risk of endocarditis in the presence of bacteremia. ${ }^{20}$

Prophylaxis against infective endocarditis is reasonable
for the following patients who undergo dental
procedures that involve manipulation of either gingival
tissue or the periapical region of teeth or perforation of
the oral mucosa*
Patients with prosthetic cardiac valve or prosthetic
material used for cardiac valve repair.
Patients with previous infective endocarditis.
Patients with CHD both unrepaired and repaired during
the first 6 months after procedure.
Cardiac transplant recipients with valve regurgitation
due to a structurally abnormal valve.
*Prophylaxis against infective endocarditis is not recommended
for non-dental procedures in the absence of active infection.

Table 5: Antibiotic Prophylaxis for infective endocarditis in high-risk patients.

ACOG (20Ila)
Standard (IV): ampicillin $2 \mathrm{~g}$ or cefazolin or ceftriaxone
$1 \mathrm{~g}$
Penicillin-allergic (IV): cefazolin or ceftriaxone* $1 \mathrm{~g}$ or
clindamycin† $600 \mathrm{mg}$
Oral: amoxicillin $2 \mathrm{~g}$
American Heart Association (Wilson, 2007):
Standard: ampicillin 2 g IV or IM or amoxicillin $2 \mathrm{~g}$ PO
Penicillin-allergic: clarithromycin or azithromycin 500
mg PO; cephalexin 500 mg PO; clindamycin $600 \mathrm{mg}$
PO, IV, or IM; or cefazolin or ceftriaxone $1 \mathrm{~g}$ IV or IM
*This regimen does not cover enterococcus. Vancomycin can be
used if enterococcus is of concern.
$\dagger$ Cephalosporins should not be used in patients with a
significant sensitivity to penicillins.

\section{$2^{\text {nd }}$ stage of labor}

Women usually deliver easily; vacuum/outlet forceps may be used to cut short the second stage whenever required.

\section{$3^{\text {rd }}$ stage of labor}

Follow active management of the third stage of labour (AMTSL) guidelines. In case of Primary postpartum haemorrhage $(\mathrm{PPH})$, concentrated oxytocin infusion, IM carboprost or Misoprost per-rectum can be given.

\section{After delivery of baby}

- Patient to be put in sitting position with legs hanging down to aid peripheral pooling. 
- Constant vigilance for signs of failure.

- Inj. lasix 20mg/40mg I/V bolus dose to be given. (Provided there was no $\mathrm{PPH}$ and $\mathrm{BP}>110$ systolic).

- Observation in labor room for 12 hours.

7. Post-partum care

- Not to be discharged before one week.

- Watchful expectancy for 1st 48 -72 hours postdelivery for S/S of pulmonary edema. ${ }^{21,22}$

- Antibiotics to continue for 7 days after delivery

- Breast feeding to continue, except in Class III,IV.

\section{Contraception}

- OC pills, barrier contraceptives- not recommended. ${ }^{23}$

- IUCDs can be used in uncomplicated heart disease

- Progestin only pills (Desogestral) or Long acting injectable progesterone are better (Medroxy progesterone 150mg IM every 3 months)

- Permanent Sterilization (by Minilap/partner's vasectomy) is best and considered if family is complete.

9. Follow-up

- At 6 weeks.

- To the cardiologist as indicated according to the functional status of the patient.

\section{Management of pulmonary edema}

- Advise arterial blood gases (ABG), Call to medical specialist/ ICU.

- Patient to be postured in sitting position.

- Intranasal Oxygen administration to raise SPO2 $>60 \%$.

- Assisted mechanical ventilation required if $\mathrm{SPO} 2<60 \%$.

- IV Injection Morphine 2-5mg (15mg /ml) given over 5 minutes.

- IV Furosemide (Lasix) 20-40 mg slowly, repeated every 15-30 minutes till response/ $\mathrm{BP}<$ $90 \mathrm{~mm} \mathrm{Hg} /$ maximum $200 \mathrm{mg}$.

\section{Management of specific diseases}

1. Recommendations for the management of valvular heart disease 2

a) Mitral stenosis(MS)

- In patients with symptoms or pulmonary hypertension, restricted activities and $\beta 1$-selective blockers are recommended.24,25
- Diuretics are endorsed when congestive symptoms persist despite $\beta$-blockers. 25

- Patients with severe MS should undergo intervention pre-pregnancy.

- Therapeutic anticoagulation is recommended in atrial fibrillation, left atrial thrombosis, or prior embolism.

- Percutaneous mitral commissurotomy should be considered in pregnant patients with severe symptoms or systolic pulmonary artery pressure $>50$ $\mathrm{mmHg}$ despite medical therapy.

- b) Aortic stenosi(AS)

- Patients with severe AS should undergo intervention pre-pregnancy if they are symptomatic8 or LV dysfunction ( $\mathrm{LVEF}<50 \%$ ) is present

- Asymptomatic patients with severe AS should undergo intervention pre-pregnancy when they develop symptoms or have a fall in blood pressure below baseline during exercise testing.

c) Regurgitant lesions

- Patients with severe aortic or mitral regurgitation and symptoms or impaired ventricular function or ventricular dilatation should be treated surgically pre-pregnancy.

- Medical therapy is recommended in pregnant women with regurgitant lesions when symptoms occur.

\section{d) Mechanical valves}

- Oral Anticoagulants (OAC) are recommended during second and third trimesters until the 36th week.

- Change of anticoagulation regimen during pregnancy should be implemented in hospital.

- If delivery starts while on OACs, caesarean delivery is indicated.

- $\quad$ OAC should be discontinued and dose-adjusted Unfractionated heparin (UFH) (a PTT $\geq 2 \times$ control) or adjusted-dose low molecular weight heparin (LMWH) (target anti-Xa level 4-6 hours post-dose $0.8-1.2 \mathrm{U} / \mathrm{mL}$ ) started at the 36 th week of gestation.

- In pregnant women managed with LMWH, the post-dose anti-Xa level should be assessed weekly.

- LMWH should be replaced by intravenous UFH at least 36 hours before planned delivery. UFH should be continued until 4-6 hours before planned delivery and restarted 4-6 hours after delivery if there are no bleeding complications.

- Immediate echocardiography is indicated in women with mechanical valves presenting with dyspnea and/or an embolic event.

- Continuation of OACs should be considered during first trimester if warfarin dose required for 
therapeutic anticoagulation is $<5 \mathrm{mg}$ after patient's informed consent.

- Discontinuation of OAC between weeks 6 and 12 and replacement by adjusted-dose UFH (a PTT $\geq 2 \times$ control; in high risk patients applied as intravenous infusion) or LMWH twice daily (with dose adjustment according to weight and target anti-Xa level 4-6 hours post-dose $0.8-1.2$ $\mathrm{U} / \mathrm{mL}$ ) should be considered in patients with a warfarin dose requirement of $>5 \mathrm{mg} /$ day.

- Discontinuation of OACs between weeks 6 and 12 and replacement by UFH or LMWH under strict dose control (as described above) may be considered on an individual basis in patients with warfarin dose required for therapeutic anticoagulation $<5 \mathrm{mg}$ /day.

- Continuation of OACs may be considered between weeks 6 and 12 in patients with a warfarin dose required for therapeutic anticoagulation $>5 \mathrm{mg} /$ day

- $\quad$ LMWH should be avoided, unless anti-Xa levels are monitored.

\section{Recommendations for the management of congenital heart disease $e^{2}$}

- Pre-pregnancy relief of stenosis (usually by balloon valvulotomy) should be performed in severe pulmonary valve stenosis (peak Doppler gradient $>64 \mathrm{mmHg}$ ).

- Individual follow-up schedules should be arranged; ranging from twice during pregnancy to monthly.

- Symptomatic patients with Ebstein's anomaly with cyanosis and/or heart failure should be treated pre-pregnancy or counselled against pregnancy.

- In asymptomatic women with a severely dilated right ventricle or symptomatic women with marked dilatation of right ventricle due to severe pulmonary regurgitation, pre-pregnancy pulmonary valve replacement (bioprosthesis) should be performed

- All women with a bicuspid aortic valve should undergo imaging of the ascending aorta before pregnancy, and surgery should be considered when the aortic diameter is $>50 \mathrm{~mm}$.

- Anticoagulation treatment should be considered during pregnancy in Fontan patients.

- In PAH, associated anticoagulant treatment should be considered in patients with suspicion of pulmonary embolism as the cause (or partly the cause) of PAH.

- In patients who are already taking drug therapy for PAH before becoming pregnant, continuation should be considered after information about teratogenic effects.
- Women with pulmonary hypertension or with an oxygen saturation below $85 \%$ should be advised against pregnancy.

- Patients with TGA and a systemic right ventricle with more than moderate impairment of RV function and/or severe TR should be advised against pregnancy.

- Fontan patients with depressed ventricular function and/or moderate to severe atrioventricular valvular regurgitation or with cyanosis or with protein-losing enteropathy should be advised against pregnancy.

\section{Recommendations for the management of aortic disease $^{2}$}

- Women with Marfan syndrome or other known aortic disease should be counselled about the risk of aortic dissection during pregnancy and recurrence risk for the offspring.

- Imaging of entire aorta (CT/MRI) should be performed before pregnancy in patients with Marfan syndrome or other known aortic disease.

- Women with Marfan syndrome and an ascending aorta $>45 \mathrm{~mm}$ should be treated surgically pre-pregnancy.

- In pregnant women with known aortic dilatation, (history of) type $\mathrm{B}$ dissection or genetic predisposition for dissection strict blood pressure control is recommended.

- Repeated echocardiographic imaging every 4-8 weeks should be performed during pregnancy in patients with ascending aorta dilatation.

- For imaging of pregnant women with dilatation of the distal ascending aorta, aortic arch or descending aorta, MRI (without gadolinium) is recommended.

- In women with a bicuspid aortic valve imaging of the ascending aorta is endorsed.

- In patients with an ascending aorta $<40 \mathrm{~mm}$, vaginal delivery is favored.

- Women with aortic dilatation or (history of) aortic dissection should deliver in a center where cardiothoracic surgery is available

- In patients with an ascending aorta $>45 \mathrm{~mm}$, caesarean delivery should be considered.

- Surgical treatment pre-pregnancy should be considered in women with aortic disease associated with a bicuspid aortic valve when the aortic diameter is $>50 \mathrm{~mm}$ (or $>27 \mathrm{~mm} / \mathrm{m} 2$ BSA).

- Prophylactic surgery should be considered during pregnancy if aortic diameter is $\geq 50 \mathrm{~mm}$ and increasing rapidly. 
- In Marfan, and other patients with an aorta < 40-45 mm, vaginal delivery with epidural anesthesia and expedited second stage should be considered.

- In Marfan, and other patients with an aorta $\geq 40$ $45 \mathrm{~mm}$, caesarean section may be considered.

- Patients with (or history of) type B dissection should be advised against pregnancy.

4. Recommendations for the management of coronary artery disease ${ }^{2}$

- $\quad$ ECG and troponin levels should be performed in case of chest pain in a pregnant woman.

- Coronary angioplasty is the preferred reperfusion therapy for ST-elevation myocardial infarction during pregnancy.

- A conservative management should be considered for non ST-elevation Acute Coronary Syndrome without risk criteria.

- An invasive management should be considered for non ST-elevation ACS with risk criteria (including Non ST-elevation myocardial infarction).

5. Recommendations for the management of cardiomyopathies. ${ }^{2}$

- Anticoagulation is recommended in patients with intracardiac thrombus detected by imaging or with evidence of systemic embolism.

- Women with HF during pregnancy should be treated according to current guidelines for nonpregnant patients, respecting contraindications for some drugs in pregnancy

- Women with DCM should be informed about the risk of deterioration of the condition during gestation and peripartum.

- In patients with a past history or family history of sudden death, close surveillance with prompt investigation is recommended if symptoms of palpitations or presyncope are reported.

- Therapeutic anticoagulation with LMWH or vitamin $\mathrm{K}$ antagonists according to stage of pregnancy is recommended for patients with atrial fibrillation.

- Delivery should be performed with $\beta$-blocker protection in women with HCM.

- $\quad \beta$-blockers should be considered in all patients with HCM and more than mild LVOTO or maximal wall thickness $>15 \mathrm{~mm}$ to prevent sudden pulmonary congestion.

- In HCM, cardioversion should be considered for persistent atrial fibrillation. Due to high metabolic demands of lactation and breastfeeding, preventing lactation may be considered in PPCM.

- Subsequent pregnancy is not recommended if LVEF does not normalize in women with PPCM.

\section{Funding: No funding sources \\ Conflict of interest: None declared \\ Ethical approval: Not required}

\section{REFERENCES}

1. Simpson LL. Maternal cardiac disease: update for the clinician. Obstet Gynecol. 2012;119:345

2. The Task Force on the management of cardiovascular diseases during pregnancy of the European Society of Cardiology (ESC).ESC guidelines. European Heart Journal. 2011:32;314797.

3. Stangl V, Schad J, Gossing G, Borges A, Baumann G, Stangl K. Maternal heart disease and pregnancy outcome: a single-centre experience. Eur J Heart Fail. 2008;10:855-60.

4. Siu SC, Sermer M, Colman JM, Alvarez AN, Mercier LA, Morton BC, et al. Prospective multicenter study of pregnancy outcomes in women with heart disease.Circulation.2001;104:515-21.

5. Pearson GD, Veille JC, Rahimtoola S, Hsia J, Oakley CM, Hosenpud JD, Ansari A, Baughman KL. Peripartum cardiomyopathy: National Heart, Lung and Blood Institute and Office of Rare Diseases (National Institutes of Health) workshop recommendations and review.JAMA.2000; 283:1183-8.

6. Cunningham FG, Leveno KJ, Bloom SL, Spong YC, Dashe SJ. Cardiovascular Disorders. William's Obstetrics. 24 ${ }^{\text {th }}$ edition. McGraw Hill Education; 2014:973-99.

7. Robson SC, Dunlop W, Moore M, Hunter S. Combined Doppler and echocardiographic measurement of cardiac output: theory and application in pregnancy. Br J Obstet Gynaecol. 1987;94:1014-27.

8. Gandhi M, Martin SR. Cardiac disease in pregnancy. Obstetrics and Gynecology clinics. 2015;42(2):315-33.

9. ACOG Committee Opinion. Number 299, September 2004 (replaces No. 158, September 1995). Guidelines for diagnostic imaging during pregnancy. Obstet Gynecol. 2004;104:647-51.

10. Savu O, Jurcuţ R, Giusca S, Van MT, Gussi I, Popescu BA, Ginghina C, Rademakers F, Deprest J, Voigt JU. Morphological and functional adaptation of the maternal heart during pregnancy. Circ Cardiovasc Imaging. 2012;5(3):289-97.

11. Vitarelli A, Capotosto L.Role of echocardiography in the assessment and management of adult congenital heart disease in pregnancy. Int $\mathrm{J}$ Cardiovasc Imaging. 2011;27:843. 
12. Shellock FG, Crues JV. MR procedures: biologic effects, safety, and patient care.Radiology. 2004;232:635-52.

13. De Wilde JP, Rivers AW, Price DL. A review of the current use of magnetic resonance imaging in pregnancy and safety implications for the fetus. Prog Biophys Mol Biol.2005;87:335-53.

14. Kanal E, Barkovich AJ, Bell C, Borgstede JP, Bradley WG Jr, Froelich JW, et al. ACR guidance document for safe MR practices. AJR Am J Roentgenol. 2007;188:1447-74.

15. Van HKH, Kitsis RN, Katz SD, Factor SM. Peripartum versus idiopathic dilated cardiomyopathy in young women- a comparison of clinical, pathologic and prognostic features. Int $\mathrm{J}$ Cardiol. 1993;40:57-65.

16. Seshadri S, Oakeshott P, Nelson-Piercy C, Chappell L. Prepregnancy care. Clinical Review. BMJ. 2012;344:3467.

17. Clark SL, Hankins GD. Preventing maternal death: 10 clinical diamonds. Obstet Gynecol.2012;119:360.

18. Jastrow N, Meyer P, Khairy P, Mercier LA, Dore A, Marcotte F. Prediction of complications in pregnant women with cardiac diseases referred to a tertiary Center.Int J Cardiol. 2010;151(2):209-13.
19. Oron G, Hirsch R, Ben HA, Hod M, Gilboa Y, Davidi O, Bar J. Pregnancy outcome in women with heart disease undergoing induction of labour. BJOG. 2004;111(7):669-75.

20. ACOG practice bulletin 120, June 2011- Use of Prophylactic Antibiotics in Labor and Delivery

21. Keizer JL, Zwart JJ, Meerman RH, Harinck BI, Feuth HD, Van RJ. Obstetric intensive care admission: a 12-year review in a tertiary care centre. Eur J Obstet Gynecol Reprod Biol. 2006;128:152-6.

22. Zeeman GG: Obstetric critical care: a blueprint for improved outcomes. Crit Care Med. 2006;34:208.

23. WHO Medical eligibility criteria wheel for contraceptive use 2015.

24. Vahanian A, Baumgartner H, Bax J, Butchart E, Dion R, Filippatos G, et al. Guidelines on the management of valvular heart disease: the task force on the management of valvular heart disease of the European Society of Cardiology. Eur Heart J.2007; 28:230-68.

25. Elkayam U, Bitar F. Valvular heart disease and pregnancy part I: native valves. J Am Coll Cardiol. 2005;46:223-30.

Cite this article as: Marwah S, Sharma M, Gaikwad HS, Mohindra R. Cardiac Disease in pregnancy: still an arduous conundrum for the obstetrician. Int $\mathrm{J}$ Reprod Contracept Obstet Gynecol 2016;5:1292-9. 